\title{
Acute Effect of Different Patterns of Exercise on Mood, Anxiety and Cortical Activity
}

\author{
Thiago Teixeira Guimaraes ${ }^{1, *}$; Bruno Macedo da Costa ${ }^{1}$; Lucenildo Silva Cerqueira ${ }^{2}$; Allan \\ de Carlo Andrade Serdeiro ${ }^{1}$; Fernando Augusto Monteiro Saboia Pompeu ${ }^{1}$; Helena Sales de \\ Moraes ${ }^{3}$; Tony Meireles dos Santos ${ }^{4}$; Andrea Camaz Deslandes ${ }^{3}$ \\ ${ }_{2}^{3}$ School of Physical Education and Sports, Federal University of Rio de Janeiro, Rio de Janeiro, Brazil \\ ${ }_{3}^{2}$ Department of Biomedical Engineering Program, Federal University of Rio de Janeiro, Rio de Janeiro, Brazil \\ ${ }^{3}$ Institute of Psychiatry of the Federal University of Rio de Janeiro, Rio de Janeiro, Brazil \\ ${ }^{4}$ Department of Physical Education, Federal University of Pernambuco, Recife, Brazil \\ ${ }^{*}$ Corresponding author: Thiago Teixeira Guimaraes, School of Physical Education and Sports, Federal University of Rio de Janeiro, Rua Ney Armando Meziat, 71, Rio de Janeiro, Brazil. \\ Tel: +55-214109-1518, Fax: +55-2133936055, E-mail: thiagotguimaraes@yahoo.com.
}

Received: March 5, 2014; Revised: March 8, 2014; Accepted: March 16, 2014

\begin{abstract}
Background: Nowadays, one of the most important challenges of human beings is physical inactivity. There is evidence that a positively behavioral response, modulated by a single period of exercise can predict the level of engagement during the next six to 12 months.

Objectives: The aim of this study was to investigate the effects of three different combinations of intensity and duration of effort, on a cycle ergometer, on mood, anxiety and cortical activity of physically active young males.

Materials and Methods: Ten men, aged 20 to 27 years, underwent three experimental conditions randomly (submaximal, maximal and supramaximal effort). Immediately before and after the treatments, behavior was evaluated by the Profile of Mood States(POMS) and Trait Anxiety Inventory (STAI ) scales. Standardized low resolution brain electromagnetic tomography (sLORETA) was performed to investigate the cortical activity.

Results: While submaximal effort reduced total mood disturbance, more intense efforts (maximal and supramaximal) worsened the $\operatorname{mood}(\mathrm{P}=0.001)$. This result was more prominent in supramaximal effort. Anxiety was increased in the supramaximal effort compared to others $(\mathrm{P}=0.006)$, which was not significantly different. The sLORETA analysis revealed a significant increase in high alpha and beta activity in the parahippocampal gyrus of the limbic lobe, related to brain reward system, after maximal effort $(\mathrm{P} \leq 0.05)$.

Conclusions: Acute changes in mood, anxiety and cortical activity are dependent on exercise intensity. Physically active young males, but not athletes showed positive results on subjective or neurobiological behavioral variables in submaximal and maximal efforts, respectively. This investigation could be useful to promote adherence to regular physical activity.
\end{abstract}

Keywords:Motor Activity; Electroencephalography; Behavior

\section{Background}

Long-term physical exercise is an important method to promote health with relatively low cost (1-4). However, thousands of people die annually from diseases caused by physical inactivity worldwide $(5,6)$. One of the greatest current challenges is to promote adherence to physical activity $(7,8)$. There is supporting evidence that behavioral response, mediated by a single bout of exercise, predicts participants engagement during the next six to 12 months (9). Therefore, the term adherence to exercise refers to chronic issues; the study of acute effect of different courses of exercise on subjective and neurobiological behavioral variables becomes relevant.

According to Ekkekakis and Petruzzello (10), there is a
U-shaped association between aerobic exercise intensity and affective state, characterized by the interaction of behavioral factors like happiness, euphoria, relaxation and stress reduction. This theory suggests that the optimal effort intensity with the most positive affective response would be a moderate one that is near the ventilatory threshold ( $\left.65 \% \mathrm{VO}_{2 \max }\right)$ (11). However, a meta-analysis conducted by Reed and Ones (12) showed that low-intensity exercises with a duration of 35 minutes or less induced a strong activation of positive affect. Contrarily, recent researches have shown that an exercise protocol, including high-intensity intervals generated more pleasure than a program with continuous stimulus of moder-

Implication for health policy/practice/research/medical education:

Physical inactivity is one of leading cause of chronic non-communicable diseases. One of the biggest challenges today has been decreasing rates of physical inactivity. There is evidence that a positively behavioral response modulated by a single bout of exercise can predict the level of engagement in periods of six to 12 months later. Subjective and neurobiological behavioral variables modulated by different intensities of exercise may be useful in the context of adherence to regular physical activity.

Copyright (c) 2015, Tehran University of Medical Sciences. This is an open-access article distributed under the terms of the Creative Commons Attribution License, which permits unrestricted use, distribution, and reproduction in any medium, provided the original work is properly cited. 
ate intensity (13). There is also evidence suggesting that positive behavioral outcomes tend to occur after exercise at a self-selected intensity (14). Although, intensity seems to be the main regulator of variable affective responses, different results are found in the literature. Observing more objective variables can contribute to a broader discussion on the issue.

Various techniques like functional magnetic resonance imaging (fRMI), positron emission tomography (PET), single photon emission tomography (SPECT) and electroencephalography (EEG) are used to study possible changes in brain activity in humans. Standardized lowresolution brain electromagnetic tomography (sLORETA) is another method which represents a novel EEG approach, allowing bioelectrical location determination of three-dimensional signals and not just restricted cortical events (15). Mathematical models used by sLORETA are able to estimate the intracerebral volume and infer the specific activity of neuro-anatomical structures like Brodmann areas (BA). Although, this technique is economic and noninvasive with good spatial and temporal resolution compared to fMRI and PET (16-18), however, few studies used this method in the field of exercise, worldwide. Schneider et al. (19) found greater activity in areas associated with brain reward system, doing the preferred effort (treadmill) and cycle ergometer, in 11 recreational young runners who underwent three different types of maximal test (treadmill, cycle ergometer and upper body ergometer). In another study by the same authors on 22 recreational runners aged 21 to 45 years, activation of areas related to positive emotions was found (20). However, no behavioral subjective scales were applied in such studies. In our laboratory, we found greater activity in areas related to the integration of affective and cognitive information in 19 active young male, along with a reduction in total mood disturbance, followed by a 20-minute moderate effort condition at $80 \%$ heart rate reserve (21).

\section{Objectives}

The objective of this study was to investigate the acute response of submaximal, maximal and supramaximal efforts, on a cycle ergometer, on subjective states of mood and anxiety, and cortical activity of physically active young males. The sLORETA is a very modern technique in the area of exercise, which can contribute to further discussions on cortical phenomena, associated with subjective behavioral variables. Our hypotheses were; 1 ) Moderate exercise improves behavior, unlike very intense efforts and 2) Changes in subjective mood and anxiety are accompanied by specific changes in cortical activity.

\section{Materials and Methods}

\subsection{Sample}

We recruited 10 healthy men, practicing regular physi- cal activity (at least one-hour sessions of aerobic, muscular strength and endurance exercises three times a week). Their age, weight and height ranged between 20 and 27 years, 62 and 82.5 kilograms and 1.66 and 1.85 meters, respectively. Those who were illiterate, colorblind, smoker, with mental illnesses or using any psychoactive substances (like antidepressants and anxiolytics) were excluded. One subject was excluded because of not completing the maximal effort protocol.

\subsection{Experimental Procedure}

Participants received written information about adopted routines, procedures and possible risks, through the consent term. This study was approved by the Ethics Committee of the Universidade Gama Filho (protocol number 172.2011). All volunteers were instructed not to consume stimulants and central nervous system depressants, like foods containing caffeine or alcohol, do not engage in strenuous exercise in 48 hours before the tests, not eating within three hours prior to interventions and sleep for at least seven hours the night before the tests. An anamnesis was applied prior to each protocol, to verify that all instructions were followed and whether there were other important changes.

The subjects performed three different combinations of intensity and duration of exercises on a cycle ergometer (Monark®, Brazil) including submaximal, maximal and supramaximal. The treatments were randomized with one-week intervals and always at the same time of day. Wasserman test was adopted for maximal effort (22). After three minutes of warming-up, there was a gradual increase in workload $\left(10 \% \mathrm{VO}_{2 \max }\right)$ under a fixed rate (by using a metronome audiovisual), according to body weight and age (23) at each stage of one minute. Oxygen uptake (VO2), carbon dioxide output (VCO2), minute ventilation (VE) and respiratory exchange ratio (RER)were determined by indirect calorimetry (Vista Mini-CPX®, VacuMed®, Ventura, CA, USA). The subjects used a pneumotachograph (Hans Rudolph Inc®, Kansas City, MO, USA) inserted in a turbine with bidirectional gas flow (MIR Turbocharge®, VacuMed®, Ventura, CA, USA). Before each test, ergospirometer was calibrated in a closed loop. Perceived exertion was measured using 6- to 20-point Borg Scale. The test was stopped in case of three of the following criteria: $\mathrm{VO}_{2}$ plateau $\leq 150 \mathrm{~mL} / \mathrm{min}$ or $2 \mathrm{~kg}, \mathrm{~mL} /$ min, heart rate $\geq 90 \%$ predicted by age (220-age), Borg Scale $\geq 18$, RER $\geq 1.15$ and voluntary failure to maintain the cadence.

The submaximal effort went on for 30-62 minutes $\pm 9 \%$ $\mathrm{VO}_{2 \text { max }}$. This intensity was determined from the anaerobic threshold, obtained by two experienced researchers through the respiratory equivalent (EQV), (11) and simplified V-slope methods (24). The EQV was defined as the moment when the ventilatory equivalent for oxygen consumption $\left(\mathrm{VE} / \mathrm{VO}_{2}\right)$ increased, without a concomitant increase in the ventilatory equivalent for the excretion of 


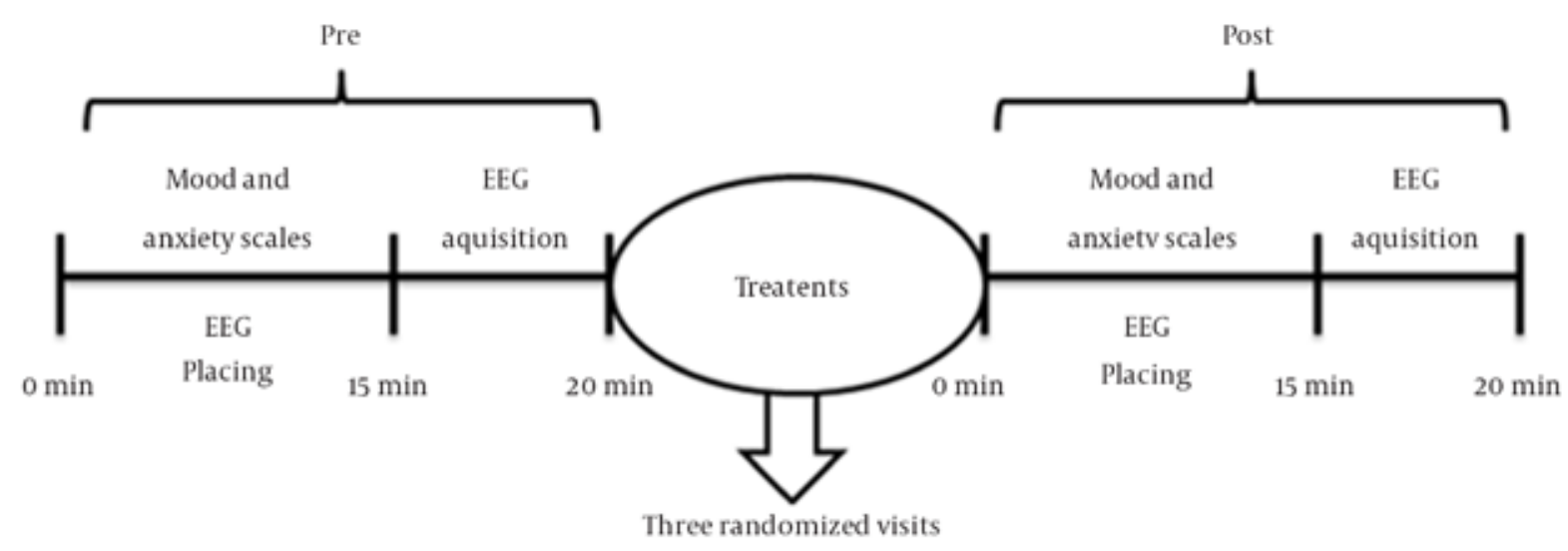

Figure 1. Experimental Design of the Study

carbon dioxide $\left(\mathrm{VE} / \mathrm{VCO}_{2}\right)$. [The EQV is the moment when there is an increase of the ventilatory equivalent for oxygen consumption $\left(\mathrm{VE} / \mathrm{VO}_{2}\right)$ without the concurrent increase in the ventilatory equivalent for carbon dioxide excretion $\left(\mathrm{VE} / \mathrm{VCO}_{2}\right)$ ]. The simplified V-slope method was examined in a Cartesian coordinate graph, with $\mathrm{VO}_{2}$ in the abscissa axis and $\mathrm{VCO}_{2}$ in the ordinate axis. The $\mathrm{VO}_{2}$ at the anaerobic threshold was determined as the breakpoint in the plot of $\mathrm{CO}_{2}$ as a function of $\mathrm{VO}_{2}$, where the slope becomes $>1$ by the simplified V-slope method (24). The load and the cadence remained stable during the whole test using an audio-visual metronome. Respiratory gases and perceived exertion sensations were checked following the same procedures described above. The supramaximal effort consisted of 30 seconds sprint against a workload corresponding to $0.075 \mathrm{kp}$ body mass $\left(\mathrm{kg}^{-1}\right)$ (25). [The subjects were instructed to pedal progressively for about five seconds to allow the workload to be adjusted manually and to remove the influence of inertia on the performance tests]. During 30 seconds, the subjects were verbally encouraged to pedal as fast as possible. The number of revolutions per minute was used to calculate the load in Watts obtained through a manual counter. The Borg Scale was used to check the perceived exertion sensations immediately after exercise.

The behavioral and cortical variables were analyzed before and immediately after the treatments. We used Profile of Mood State (POMS) (26) and State-Trait Anxiety Inventory (STAI) (27) scales to assess mood and anxiety, respectively. Assessment of cortical activity is described below. Figure 1 illustrates the experimental design of this study.

\subsection{Acquisition, Reduction and Analysis of Electro- encephalographic Signals (EEG)}

EEG was recorded for five minutes in a relaxed sedentary position from 20 monopolar electrode sites (Fz, Cz, Pz, Oz, Fp1, Fp2, F3, F4, F7, F8, C3, C4, T3, T4, T5, T6, P3, P4, $\mathrm{O} 1, \mathrm{O} 2$ ). International 10/20 system (referred to linked earlobes) was used for electrode placement with a Brain-tech
3000 (EMSA-Medical Instruments, Brazil). Eye-movement artifact was monitored with a bipolar electrode montage, using two $9 \mathrm{~mm}$ diameter electrodes, attached superiorly to and on the external canthus of the right eye. Following digital filters were used: notch $(60 \mathrm{~Hz})$, high-pass of 0.16 $\mathrm{Hz}$ and low-pass of $35 \mathrm{~Hz}$. Data was digitized at $240 \mathrm{~Hz}$, with a 12-bit resolution. We used the linked ears reference given that this is the routine in our laboratory. After that an average reference was used a posteriori.

EEG data was analyzed using sLORETA, in which the image represents the standardized electric activity of each voxel in neuro-anatomic Montreal Neurological Institute (MNI) space as the exact magnitude of the estimated current density. Anatomical labels like Brodmann areas, are also reported using MNI space, with correction to the Talairach space (28). The intracerebral volume is partitioned in 6239 voxels at $5 \mathrm{~mm}$ spatial resolution. A minimum of 120 epochs of artifact-free resting EEG were exported for further analysis using the sLORETA software provided by the KEY Institute for Brain-Mind Research (University Hospital of Psychiatry, Zurich, Switzerland; http://www.uzh.ch/Keyinst/NewLORETA/LORTA01.htm). Next, data was log-transformed and calculated in delta, theta, alpha and beta activity for each subject in each treatment. Cross-spectra of each subject and for each frequency band were transformed to sLORETA files by using the sLORETA transformation matrix.

\subsection{Statistical Analysis}

Friedman nonparametric test and Wilcoxon were used for POMS and STAI deltas values (post-pre exercise) to compare the differences between treatments. The sLORETA software was used to analyze EEG activity. After a critical threshold was defined ( $t$ critical), voxels with statistical values exceeding this threshold were accepted. The omnibus hypothesis (that all the voxel hypotheses are true) was rejected if a voxel value exceeded the critical threshold for $\mathrm{P}<0.05$ defined by 5000 randomizations. Voxel-by-voxel $\mathrm{t}$ values in Talairach space were displayed as Statistical Parametric Maps (SPMs). 
Teixeira Guimaraes Tet al.

Table 1. Comparisons of Scores of the Mood (POMS) and Anxiety (STAI) Scales. Friedman Nonparametric Test Followed by Wilcoxon Test $\mathrm{t}^{\mathrm{a}, \mathrm{b}}$

\begin{tabular}{|c|c|c|c|c|c|c|}
\hline Variables & Pre & Post & Delta & $\mathrm{X}^{2}$ & $\mathbf{P}$ & Wilcoxon \\
\hline Tension & - & - & - & 4.514 & 0.105 & - \\
\hline Sub & $3(3 ; 5)$ & $3(2 ; 4)$ & $0(-2 ; 0)$ & - & - & - \\
\hline Max & $3(2 ; 5)$ & $5(3 ; 6)$ & $1(0 ; 3)$ & - & - & - \\
\hline Supra & $4(3 ; 4)$ & $6(4 ; 7)$ & $2(0 ; 4)$ & - & - & - \\
\hline Depression & - & - & - & 4.846 & 0.089 & - \\
\hline Sub & $0(0 ; 1)$ & $0(0 ; 0)$ & $0(-1 ; 0)$ & - & - & - \\
\hline Max & $0(0 ; 1)$ & $0(0 ; 2)$ & $0(0 ; 1)$ & - & - & - \\
\hline Supra & $0(0 ; 1)$ & $0(0 ; 2)$ & $0(0 ; 1)$ & - & - & - \\
\hline Hostility & - & - & - & 3.556 & 0.169 & - \\
\hline Sub & $0(0 ; 1)$ & $0(0 ; 0)$ & $0(-1 ; 0)$ & - & - & - \\
\hline Max & $0(0 ; 0)$ & $0(0 ; 1)$ & $0(0 ; 1)$ & - & - & - \\
\hline Supra & $0(0 ; 0)$ & $1(0 ; 2)$ & $0(0 ; 2)$ & - & - & - \\
\hline Vitality & - & - & - & 3.706 & 0.157 & - \\
\hline Sub & $12(10 ; 16)$ & $16(11 ; 17)$ & $1(-1 ; 3)$ & - & - & - \\
\hline Max & $14(12 ; 18)$ & $14(7 ; 15)$ & $-1(-7 ; 2)$ & - & - & - \\
\hline Supra & $14(11 ; 17)$ & $11(4 ; 17)$ & $-2(-6 ; 0)$ & - & - & - \\
\hline Fatigue & - & - & - & 4.941 & 0.085 & - \\
\hline Sub & $1(1 ; 3)$ & $3(1 ; 4)$ & $0(-1 ; 3)$ & - & - & - \\
\hline Max & $3(3 ; 5)$ & $13(6 ; 14)$ & $6(3 ; 10)$ & - & - & - \\
\hline Supra & $2(1 ; 4)$ & $8(6 ; 16)$ & $6(3 ; 13)$ & - & - & - \\
\hline Confusion & - & - & - & 4.323 & 0.115 & - \\
\hline Sub & $4(2 ; 6)$ & $4(3 ; 5)$ & $0(0 ; 1)$ & - & - & - \\
\hline Max & $2(1 ; 5)$ & $4(3 ; 7)$ & $2(0 ; 3)$ & - & - & - \\
\hline Supra & $4(2 ; 5)$ & $7(4 ; 9)$ & $3(1 ; 4)$ & - & - & - \\
\hline Total mood disturbance & - & - & - & 13.941 & 0.001 & - \\
\hline Sub & $96(92 ; 111)$ & $92(90 ; 103)$ & $-2(-10 ; 2)$ & - & - & $\operatorname{Sub}^{*} \operatorname{Max}(\mathrm{P}=0.012)$ \\
\hline $\operatorname{Max}$ & $98(89 ; 115)$ & $107(98 ; 125)$ & $9(5 ; 12)$ & - & - & Sub*Supra $(P=0.008)$ \\
\hline Supra & $94(90 ; 104)$ & $118(106 ; 126)$ & $18(5 ; 28)$ & - & - & Supra*Max $(P=0.049)$ \\
\hline Anxiety (STAI) & - & - & - & 10.364 & 0.006 & - \\
\hline Sub & $33(29 ; 34)$ & $30(25 ; 36)$ & $1(-3 ; 1)$ & - & - & Sub*Supra $(P=0.012)$ \\
\hline Max & $33(27 ; 36)$ & $34(31 ; 44)$ & $-1(-2 ; 7)$ & - & - & - \\
\hline Supra & $29(27 ; 36)$ & $44(33 ; 48)$ & $4(2 ; 15)$ & - & - & Supra*Max $(P=0.037)$ \\
\hline
\end{tabular}

\section{Results}

The values (mean and standard deviation) of heart rate, oxygen consumption, power and perceived exertion sensations effort were $144 \pm 7 \mathrm{bpm} ; 2.48 \pm 0.37$ L.min ${ }^{-1} ; 150 \pm 26$ Watts; $13 \pm 2$ Borg (submaximal effort),

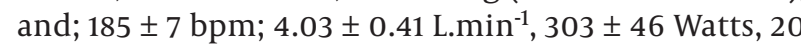
\pm 0 Borg (maximal effort). Maximal effort lasted from eight to 12 minutes. In the supramaximal treatment a power of 631 watts \pm 4 and Borg of $20 \pm 0$ were observed. The results of mood (POMS) and anxiety (STAI) are shown in Table 1 . There was a difference in total mood disturbance between all of treatments $(P=0.001)$. Submaximal effort reduced the total mood disturbance, while maximal effort increased it and supramaximal effort caused an even greater increase. The other variables of POMS did not change significantly. Regarding anxiety, there was a significant difference between submaximal and supramaximal $(\mathrm{P}=0.012)$, as between maximal and supramaximal $(\mathrm{P}=0.037)$, indicating that it is increased in supramaximal effort compared to the other two protocols, which did not differ. 


\section{Figure 2. Analysis of sLORETA.}

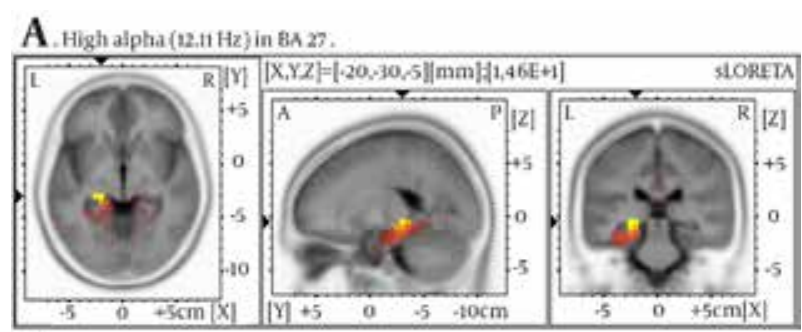

B. High alpha $(22.27 \mathrm{~Hz})$ in $\mathrm{BA} 27$,

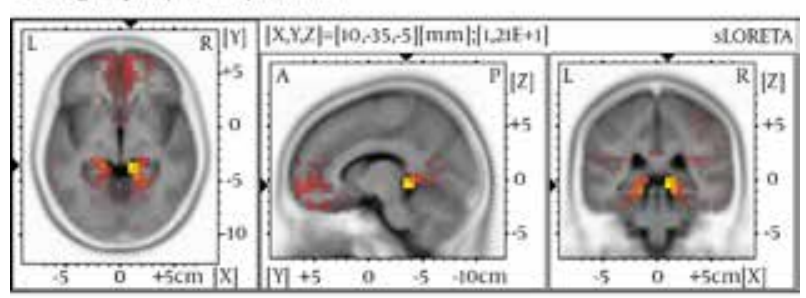

C. High alpha $(26,17 \mathrm{~Hz})$ in $\mathrm{kA} 19$.

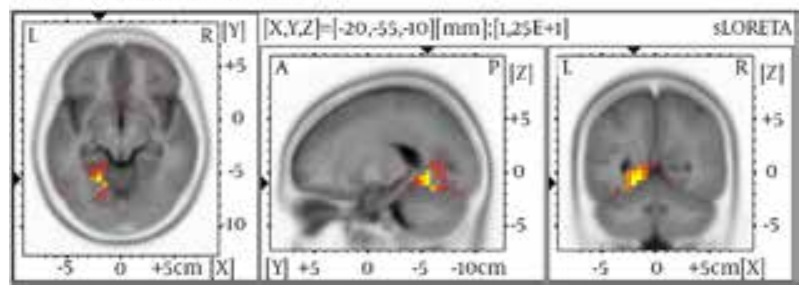

Statistical parametric maps (voxel-by-voxel) of sLORETA differences comparing pre and post moment in maximal effort, A. High alpha $(12.11 \mathrm{~Hz})$ in BA 27, B. High beta $(22.27 \mathrm{~Hz})$ in BA 27, C. High beta $(26.17 \mathrm{~Hz})$ in BA 19,19Red and yellow colors indicate significant increase in POST measurement shown for high alpha in BA 27 (A), high beta in BA 27 (B), and high beta in BA19 (C). For X (- represent left, + represent right), Y (- represent posterior, + represent anterior), Z (- represent inferior, + represent superior).

Through sLORETA analysis, significant increases in maximal effort at the post moment were seen, when compared to pre, for high alpha $(14.62 \mathrm{~Hz})$ and high beta $(12.11 \mathrm{~Hz})$ in BA 27 (parahippocampal gyrus-limbic lobe), and high beta $(12.46 \mathrm{~Hz})$ in BA 19 (parahippocampal gyrus-limbic lobe). Figure 2 illustrates the changes mediated by maximal effort. No significant differences were found between pre and post submaximal and supramaximal efforts.

\section{Discussion}

The aim of this study was to investigate acute effect of three different patterns of exercise on a cycle ergometer on mood, anxiety and cortical activity of physically active young males, through manipulation of intensity and duration of the effort. The submaximal effort reduced the total mood disturbance. The maximal effort, of short and progressive character, activated areas of the brain related to pleasure and reward, but increased total mood disturbance. The supramaximal effort increased total mood disturbance and anxiety.

We agree with the review of Ekkekakis and Petruzzello (10) which suggested greater benefits in behavioral re- sponse to acute moderate effort. Although Bartlett et al. (13) concluded that a session with intervals containing maximal stimuli is a good strategy to motivate young and physically active subjects compared to a continuous protocol, but it is too early to affirm that short-term intense stimuli can promote behavior and adherence to exercise. In the present study, we reported a worsening in mood and anxiety due to a single supramaximal effort. Richards et al. (29), following a similar protocol, reported occurrence of dizziness and nausea in some subjects. We also observed the same in three subjects.

We did not support some findings in the literature regarding other factors of the POMS. For example, Fumoto et al. (30) submitted 10 healthy physically active young (nine men and one woman) to 15 minutes at moderate intensity on a cycle ergometer and found a significant reduction of tension and confusion, and a tendency to increase vigor factor and reduce depression factor. Woo et al. (31) submitted 16 young women to moderate intensities $\left(61.4 \% \mathrm{VO}_{2 \mathrm{max}}\right)$ in three different durations $(15,30$ and 45 minutes) and found a higher score after a half hour vigor activity. We found no significant differences regarding tension, depression, hostility, vigor, fatigue and confusion in moderate effort.

sLORETA revealed an increase of high alpha and beta activity in the parahippocampal gyrus of the limbic lobe (high alpha and beta in BA 27, and high beta in BA 19) in maximal exercise. These areas are associated with reward system, pleasure, positive emotion and memory. Schneider et al. (19) submitted 11 recreational young runners to three different types of maximal effort as treadmill, cycle ergometer and upper body ergometer. The authors found that the most preferred type of effort by the subjects, induced an increase of alpha in frontal (BA 6, 8 and 9) and limbic (BA 24 and 32) lobes, as well as beta in parietal lobe (BA 7). The cycle ergometer exercise increased alpha and beta in parietal (BA 7) and limbic (AB 23 and 31). Upper body ergometer effort increased alpha in frontal lobe (BA 45) and beta in parietal lobe (BA 7 and 40). We agree with the authors because we also found changes in the limbic lobe, especially in the same type and intensity of effort. However, we did not use the cap system for electrode placement, which gives an optimization of time in recording EEG signals. After 15 minutes, the time it takes to start EEG collecting, Schneider et al. (19) reported increases of alpha in frontal (BA 6 and 9) and limbic (BA 24 and 32 ) lobes, regions associated with reward and pleasure, only in the cycle ergometer (upper body ergometer increased alpha and beta in the temporal lobes). Although they found an increase of alpha in limbic lobe, they observed no changes in beta, as we found in the present study. Another relevant aspect was the absence of subjective measures of behavior parallel to biological measure in the study of Schneider et al. (19) to allow a fuller discussion of findings.

In another study, 22 young recreational runners underwent a maximal effort on treadmill and there were sig- 
nificant increases in low alpha in the left frontal gyrus (BA 8), related to the processing of positive emotions (32). However, after 15 minutes, which represents a real comparison with our protocol, there was a reduction in high alpha, low beta and gamma in areas accounting for language (BA18, 20, 21 and 22). Moreover, Schneider et al. (32) in this study did not use subjective scales to analyze the behavior changes. Moreover, Moraes et al. (21) found significant changes in SLORETA followed by a reduction in total mood disturbance in 19 young active (11 Men and 8 Women) after 20 minutes of moderate exercise on a cycle ergometer. Increases in low alpha in the anterior cingulate cortex (BA 24), low beta in the same region (BA 33) and high beta in posterior cingulate cortex (BA 23) were observed. In this study, we observed a lower total mood disturbance after submaximal effort, but no significant electrophysiological change was observed by sLORETA.

Improvement in brain areas mediated by high intensities, especially in subjects with high level of physical fitness, could be justified by the postulates of Solomon (33). According to a study performed on a group of paratroopers about their emotional experiences, the feeling of fear to jump was followed by a very intense pleasure when the equipment is opened. The opposite feeling of fear, corresponding to the relief and pleasure, represents a resource to stabilize emotion, as well as a psychological reinforcement encouraging the repetition of action. This emotional duality has given rise to the opponent process theory or hedonistic hypothesis, later expanded to other situations, including intense physical exercise. High intensities of exercise in active subjects can promote positive reinforcement. We know that endurance and adventure athletes, or subjects with high levels of physical fitness, undergo numerous challenging sessions that cause fear or pain, repeating them probably due to "the costs of pleasure and the benefits of pain" (33). In the present study, although there was an increase in total mood disturbance immediately after maximal effort, activation of the brain reward system was verified by sLORETA. An important limitation probably explaining the difference in results between the subjective and objective variables was the time of application of the behavioral scales, which occurred immediately after the treatments, and the capture of EEG, which performed 15 minutes after the end of the sessions, time required for the placement of the electrodes. We do not know if the supramaximal effort, given the exposed context, could present some sort of neurobehavioral benefit after a long period (for example 60 minutes after the 30 seconds sprint).

We conclude that acute changes in mood, anxiety and cortical activity are dependent on the effort intensity. Physically active youth, but not athletes, show positive results on subjective or neurobiological behavioral variables in submaximal and maximal exercises. This information may be useful to promote adherence to regular physical activity.

\section{Acknowledgements}

The authors would like to thank the staff of Neuroscience Laboratory of Exercise - UFRJ.

\section{Authors' Contributions}

Study concept and design; Thiago Teixeira Guimaraes, Bruno Macedo da Costa, Lucenildo Silva Cerqueira, Andrea Camaz Deslandes; Analysis and interpretation of data: Thiago Teixeira Guimaraes, Lucenildo Silva Cerqueira, Allan de Carlo Andrade Serdeiro, Bruno Macedo da Costa; Drafting of the manuscript: Thiago Teixeira Guimaraes, Andrea Camaz Deslandes; Critical revision of the manuscript for important intellectual content: Fernando Augusto Monteiro Saboia Pompeu, Helena Sales de Moraes, Tony Meireles dos Santos, Andrea Camaz Deslandes; Statistical analysis: Thiago Teixeira Guimaraes, Lucenildo Silva Cerqueira.

\section{Financial Disclosure}

There was no financial interest.

\section{Funding/Support}

This research was supported by FAPERJ.

\section{References}

1. Booth FW, Chakravarthy MV, Spangenburg EE. Exercise and gene expression: physiological regulation of the human genome through physical activity. J Physiol. 2002;543(Pt 2):399-411.

2. Vaynman S, Gomez-Pinilla F. Revenge of the "sit": how lifestyle impacts neuronal and cognitive health through molecular systems that interface energy metabolism with neuronal plasticity. J Neurosci Res. 2006;84(4):699-715.

3. Dishman RK, Berthoud HR, Booth FW, Cotman CW, Edgerton VR, Fleshner MR, et al. Neurobiology of exercise. Obesity (Silver Spring). 2006;14(3):345-56.

4. Deslandes A, Moraes H, Ferreira C, Veiga H, Silveira H, Mouta R, et al. Exercise and mental health: many reasons to move. Neuropsychobiology. 2009;59(4):191-8.

5. Handschin C, Spiegelman BM. The role of exercise and PG C1alpha in inflammation and chronic disease. Nature. 2008;454(7203):463-9.

6. Lee IM, Shiroma EJ, Lobelo F, Puska P, Blair SN, Katzmarzyk PT. Ef fect of physical inactivity on major non-communicable diseases worldwide: an analysis of burden of disease and life expectancy. The Lancet. 2012;380(9838):219-29.

7. Bryan AD, Nilsson R, Tompkins SA, Magnan RE, Marcus BH, Hutchison KE. The Big Picture of Individual Differences in Physical Activity Behavior Change: A Transdisciplinary Approach. Psychol Sport Exerc. 2011;12(1):20-6.

8. Dasilva SG, Guidetti L, Buzzachera CF, Elsangedy HM, Krinski K, De Campos W, et al. Psychophysiological responses to selfpaced treadmill and overground exercise. Med Sci Sports Exerc. 2011;43(6):1114-24.

9. Williams DM, Dunsiger S, Ciccolo JT, Lewis BA, Albrecht AE, Marcus BH. Acute Affective Response to a Moderate-intensity Exercise Stimulus Predicts Physical Activity Participation 6 and 12 Months Later. Psychol Sport Exerc. 2008;9(3):231-45.

10. Ekkekakis P, Petruzzello SJ. Acute Aerobic Exercise and Affect. JSM. 1999;28(5):337-74.

11. Caiozzo VJ, Davis JA, Ellis JF, Azus JL, Vandagriff R, Prietto CA, et al. A comparison of gas exchange indices used to detect the anaerobic threshold. J Appl Physiol Respir Environ Exerc Physiol. 1982;53(5):1184-9. 
12. Reed J, Ones DS. The effect of acute aerobic exercise on positive activated affect: A meta-analysis. Psychology of Sport and Exercise. 2006;7(5):477-514.

13. Bartlett JD, Close GL, MacLaren DP, Gregson W, Drust B, Morton JP. High-intensity interval running is perceived to be more enjoyable than moderate-intensity continuous exercise: implications for exercise adherence. J Sports Sci. 2011;29(6):547-53.

14. Ekkekakis P. Let them roam free? Physiological and psychological evidence for the potential of self-selected exercise intensity in public health. Sports Med. 2009;39(10):857-88.

15. Pascual-Marqui RD, Michel CM, Lehmann D. Low resolution electromagnetic tomography: a new method for localizing electrical activity in the brain. INT J PSYCHOL. 1994;18(1):49-65.

16. Gamma A, Lehmann D, Frei E, Iwata K, Pascual-Marqui RD, Vollenweider FX. Comparison of simultaneously recorded [H2(15) O]-PET and LORETA during cognitive and pharmacological activation. Hum Brain Mapp. 2004;22(2):83-96.

17. Mulert C, Jager L, Schmitt R, Bussfeld P, Pogarell O, Moller HJ, et al. Integration of fMRI and simultaneous EEG: towards a comprehensive understanding of localization and time-course of brain activity in target detection. Neuroimage. 2004;22(1):83-94.

18. Bai X, Towle VL, He EJ, He B. Evaluation of cortical current density imaging methods using intracranial electrocorticograms and functional MRI. Neuroimage. 2007;35(2):598-608.

19. Schneider S, Brummer V, Abel T, Askew CD, Struder HK. Changes in brain cortical activity measured by EEG are related to individual exercise preferences. Physiol Behav. 2009;98(4):447-52.

20. Schneider S, Askew CD, Diehl J, Mierau A, Kleinert J, Abel T, et al. EEG activity and mood in health orientated runners after different exercise intensities. Physiol Behav. 2009;96(4-5):709-16.

21. Moraes H, Deslandes A, Silveira H, Ribeiro P, Cagy M, Piedade R, et al. The effect of acute effort on EEG in healthy young and elderly subjects. Eur J Appl Physiol. 2011;111(1):67-75.

22. Wasserman K, Whipp BJ, Koyl SN, Beaver WL. Anaerobic threshold and respiratory gas exchange during exercise. J Appl Physiol. 1973;35(2):236-43.
23. Nogueira FS, Pompeu FA. [Maximal workload prediction models in the clinical cardio-pulmonary effort test]. Arq Bras Cardiol. 2006;87(2):137-45.

24. Schneider DA, Phillips SE, Stoffolano S. The simplified V-slope method of detecting the gas exchange threshold. Med Sci Sports Exerc. 1993;25(10):1180-4.

25. Burgomaster KA, Hughes SC, Heigenhauser GJ, Bradwell SN, Gibala MJ. Six sessions of sprint interval training increases muscle oxidative potential and cycle endurance capacity in humans. $J$ Appl Physiol (1985). 2005;98(6):1985-90.

26. McNair D, Loor M, Droppleman L. Manual for the profile of mood states.Sand Diego, California: EDITS; 1971.

27. Spielberger C, Gorsuch R, Lushene R. Manual for strait anxiety inventory.Palo Alto: Consulting Psychologists Press;1970.

28. Brett M, Johnsrude IS, Owen AM. The problem of functional localization in the human brain. Nat Rev Neurosci. 2002;3(3):243-9.

29. Richards JC, Johnson TK, Kuzma JN, Lonac MC, Schweder MM, Voyles WF, et al. Short-term sprint interval training increases insulin sensitivity in healthy adults but does not affect the thermogenic response to beta-adrenergic stimulation.J Physiol. 2010;588(Pt 15):2961-72.

30. Fumoto M, Oshima T, Kamiya K, Kikuchi H, Seki Y, Nakatani Y, et al. Ventral prefrontal cortex and serotonergic system activation during pedaling exercise induces negative mood improvement and increased alpha band in EEG. Behav Brain Res. 2010;213(1):1-9.

31. Woo M, Kim S, Kim J, Petruzzello SJ, Hatfield BD. Examining the exercise-affect dose-response relationship: does duration influence frontal EEG asymmetry? Int J Psychophysiol. 2009;72(2):166-72.

32. Schneider S, Askew CD, Abel T, Mierau A, Struder HK. Brain and exercise: a first approach using electrotomography. Med Sci Sports Exerc. 2010;42(3):600-7.

33. Solomon RL. The opponent-process theory of acquired motivation: the costs of pleasure and the benefits of pain. Am Psychol. 1980;35(8):691-712. 